\title{
Tendencia de la mortalidad a consecuencia de asfixia por inmersión en México, 1979-2005
}

\author{
Alfredo Celis, ${ }^{1,2}$ Mario Burciaga-Torres, ${ }^{2}$ Samira Castillo, ${ }^{2}$ \\ Sergio Robles-Pinto ${ }^{2}$ y María Orozco-Valerio ${ }^{1}$
}

Forma de citar Celis A, Burciaga-Torres M, Castillo S, Robles-Pinto S, Orozco-Valerio M. Tendencia de la mortalidad a consecuencia de asfixia por inmersión en México, 1979-2005. Rev Panam Salud Publica. 2008; 24(6):422-9.

RESUMEN Objetivo. Describir la tendencia de la mortalidad a consecuencia de asfixia por inmersión en México y sus estados federativos durante el período comprendido entre 1979 y 2005.

Métodos. Se estimaron tasas de mortalidad y tendencias durante el período estudiado a partir de los datos oficiales de mortalidad en México, según la Clasificación Internacional de Enfermedades (9. ${ }^{a}$ y $10 .^{a}$ revisiones). Los resultados se analizaron por grupos de edad, sexo y año en que se registró el suceso, así como por el estado federativo y el tamaño de la localidad de residencia.

Resultados. Entre 1979 y 2005 se registraron 107319 muertes a consecuencia de asfixia por inmersión. La mortalidad en el país se redujo 65,31\% (de 6,58 por 100000 habitantes en 1979-1981 a 2,28 por 100000 habitantes en 2003-2005). Esta reducción se observó en todos los grupos de edad (desde 68,10\% en el de 5 a 9 años hasta 54,30\% en el de 1 a 4 años) y estados (desde 87,19\% en Colima hasta 36,58\% en Nayarit). En el trienio 2003-2005, la mortalidad fue relativamente mayor en hombres y niños que en mujeres y niñas (razón: 5,46; IC95\%: 5,15 a 5,80); en los grupos de edad de 1 a 4 años $(4,27$ por 100 000) y de 60 añoso más (3,37 por 100000 habitantes); en el estado de Tabasco (5,92 por 100000 habitantes); y en las poblaciones de menos de 2499 habitantes (4,03\% por 100000 habitantes).

Conclusiones. La mortalidad a consecuencia de asfixia por inmersión en México presenta una tendencia descendente, a pesar de lo cual se mantiene como un importante problema de salud pública. Es necesario profundizar en la consolidación de la información sobre las muertes accidentales por esta causa, tanto desde el punto de vista de la vigilancia epidemiológica como de las intervenciones que se deben implementar para su erradicación.

Palabras clave Ahogamiento, mortalidad, México.

1 Departamento de Salud Pública, Universidad de Guadalajara, Guadalajara, Jalisco, México. La correspondencia se debe dirigir a Alfredo Celis, Departamento de Salud Pública, Universidad de Guadalajara, Sierra Morena No. 950, edificio N, Colonia Independencia, Guadalajara, Jalisco 44340, México. Correo electrónico: alfredo_celis@ yahoo.com

2 Instituto Mexicano del Seguro Social, Guadalajara, México.
La asfixia por inmersión es un problema de salud pública que en el año 2000 causó la muerte de 449000 personas en todo el mundo (7,4 por 100000 habitantes). Estas muertes corresponden en su mayoría a hombres, menores de 15 años y a personas de países con in- gresos medios y bajos (1). En el período de 2000 a 2005, la asfixia por inmersión fue la segunda causa de muerte en México por lesiones no intencionales en niños de 1 a 4 años (entre 4,0 y 4,3 por $100000)$ y de 5 a 14 años de edad (entre $1,3$ y 1,68 por 100000$)(2)$. 
Poco se sabe de la asfixia por inmersión en México. Se ha descrito que la mortalidad por esta causa en adolescentes fue de 53,7\% entre 1979 y 1997 y en la actualidad muestra una tendencia descendente (3). En el estado de Jalisco, $50 \%$ de las muertes informadas entre 1983 y 1989 ocurrieron en aljibes, presas, lagos, ríos y canales (4) y el principal factor de riesgo identificado fue la condición socioeconómica baja (5). También se ha informado como la cuarta causa de muerte de inmigrantes ilegales al tratar de cruzar la frontera de México con los Estados Unidos de América (6) y como la segunda causa de muerte de turistas fallecidos en México (7).

Debido a su importancia como problema de salud pública es prioritario estudiar los aspectos epidemiológicos más relevantes de esta causa de muerte. El objetivo de este trabajo es describir la tendencia de la mortalidad a consecuencia de asfixia por inmersión en México y sus estados federativos durante el período comprendido entre 1979 y 2005.

\section{MATERIALES Y MÉTODOS}

Para esta investigación se utilizaron los datos oficiales de mortalidad en México correspondientes a los años de 1979 a 2005. Los datos se recaban, codifican y capturan en el Instituto Nacional de Estadística y Geografía (INEGI) y están disponibles para la consulta pública en el sitio de Internet del Sistema Nacional de Información en Salud (SINAIS) de México (2). Para este análisis solo se tomaron en cuenta los registros de muerte de residentes en México cuya causa establecida es la asfixia por inmersión. Para el período 1979-1997 se consideraron las muertes registradas con los códigos E830, E832, E910, E954, E964 y E984 de la Clasificación Estadística Internacional de Enfermedades, Traumatismos y Causas de Defunción, 9. ${ }^{\mathrm{a}}$ revisión (CIE-9) (8) y para las registradas de 1998 a 2005, las correspondientes a los códigos V90, V92, W65-W74, X71, X92 y Y21 de la Clasificación Estadística Internacional de Enfermedades y Problemas Relacionados con la Salud, $10 .^{\mathrm{a}}$ revisión (CIE-10) (9).

Para el análisis se utilizaron las siguientes variables: edad, sexo y año en que se registró el suceso, así como el estado federativo y el tamaño de la localidad de residencia. Se estimaron las tasas específicas (total de defunciones a consecuencia de asfixia por inmersión en un período de tiempo entre el total de años-persona acumulados en el mismo período) estandarizadas por la población según el Censo General de Población y Vivienda del año $2000(10,11)$. Los resultados se analizaron según siete grupos de edad: menores de 1 año, de 1 a 4 , de 5 a 9 , de 10 a 19 , de 20 a 39, de 40 a 59 y 60 años o más. El denominador de las tasas se proyectó linealmente (12) a partir de la población registrada en los censos generales de población de 1980 (13), 1990 (14) y 2000 (11), y en los conteos generales de población de 1995 (15) y 2005 (16). Las tendencias de la mortalidad según el tamaño de la localidad de residencia se trazaron después de calcular las tasas de mortalidad para cada año y de agrupar las defunciones y la población según el tamaño de la localidad en tres categorías (de 1 a 2499 habitantes, de 2500 a 19999 habitantes y de 20000 habitantes o más). En este análisis solo se tomaron en cuenta los registros de 1985 a 2005, ya que los datos anteriores no eran compatibles con los más recientes.

Las tendencias se analizaron mediante regresión lineal simple. Para el cálculo de la mortalidad por trienios (desde 1979 hasta 2005) se tomó el número total de muertes en tres años y se dividió entre el número de añospersona en ese período. Se estimó el riesgo relativo (RR) de muerte a consecuencia de asfixia por inmersión para poblaciones de 1 a 2499 y de 2500 a 19999 habitantes con relación a las poblaciones de 20000 habitantes o más y sus respectivos intervalos de confianza de $95 \%$ (IC95\%). El nivel de significación fue de $P \leq 0,05$. La descripción gráfica de la mortalidad por estado federativo se realizó mediante el pa- quete estadístico Epi Map, de Epi Info para Windows (17).

\section{RESULTADOS}

Entre 1979 y 2005 se registraron 107319 muertes a consecuencia de asfixia por inmersión en diversas circunstancias (cuadro 1). En este período se observó una tendencia descendente estadísticamente significativa (figura 1) con una pendiente promedio $\beta=-0,19$ por 100000 habitantes (IC95\%: 0,21 a $-0,18 ; P<0,001$ ). La mortalidad por esta causa pasó de 6,58 por 100000 habitantes en el trienio 1979-1981 a 2,28 por 100000 habitantes en el trienio 2003-2005, lo que representó una disminución de $65,31 \%$. Esta reducción se presentó en todo el país, aunque los cambios porcentuales variaron según el estado federativo: desde $36,58 \%$ en Nayarit hasta 87,19\% en Colima (cuadro 2). La reducción se observó en todos los grupos de edad, aunque varió de 68,10\%, en el de 5 a 9 años hasta $54,30 \%$ en el de 1 a 4 años (cuadro 3).

En general, las muertes ocurrieron con mayor frecuencia en hombres y niños que en mujeres y niñas, con una razón de 5,46 (IC95\%: 5,15 a 5,80) durante el trienio 2003-2005. Según el grupo de edad, la mayor mortalidad en el trienio 2003-2005 se observó en el grupo de 1 a 4 años, con una tasa de 4,27 por 100000 habitantes, seguido por el grupo de 60 o más años, con 3,37 (cuadro 3). Llama la atención que en los hombres y niños, el grupo de 1 a 4 años ocupó el tercer lugar después de los grupos de 60 años o más y de 20 a 29 años.

En la figura 2 se ilustra la distribución de la mortalidad a consecuencia de asfixia por inmersión en los estados del país durante el trienio 2003-2005. En ella se observa que el estado con la mayor mortalidad a consecuencia de asfixia por inmersión en el trienio 2003-2005 fue Tabasco, con 5,92 por 100000 habitantes, seguido de Chiapas $(3,84)$, Tamaulipas $(3,54)$, Nayarit $(3,50)$, Oaxaca $(3,50)$, Baja California Sur $(3,44)$, Veracruz $(3,32)$ y Sinaloa 
$(3,28)$. Llama la atención que en el estado de Tabasco se mantuvo la mayor tasa de mortalidad durante todo el período estudiado (cuadro 3).

El riesgo de morir a consecuencia de asfixia por inmersión fue mayor en las poblaciones de menor número de habitantes: 2,15 (IC95\%: 2,05 a 2,26) y 1,69 (IC95\%: 1,59 a 1,80) en las poblaciones de 1 a 2499 y de 2500 a 19 999, respectivamente, con respecto a las poblaciones de 20000 habitantes o más (cuadro 4).

La tendencia de la mortalidad por esta causa fue descendente en todas las poblaciones, tanto en las de 20000 habitantes o más - que pasaron de una tasa de 3,93 por 100000 en 1985 a 1,73 por 100000 en $2005(\beta=-0,13$, IC95\%: $-0,15$ a $-0,11 ; P<0,001)-$ como en las de 1 a 2499 habitantes -que pasaron de una tasa de 6,36 por 100000 en 1985 a 3,84 por 100000 en 2005 ( $\beta=-0,19$, IC95\%: $-0,25$ a $-0,13$; $P<0,001)$ - y de 2500 a 19999 habitantes - que pasaron de una tasa de 6,67 por 100000 en 1985 a 3,06 por 100000 en $2005(\beta=-0,43$, IC95\%: $-0,55$ a $-0,31 ; P<0,001)$.
CUADRO 1. Total de muertes a consecuencia de asfixia por inmersión registradas en México, 1979-2005

\begin{tabular}{|c|c|c|c|}
\hline Muertes registradas & Código & Frecuencia & $\%$ \\
\hline \multicolumn{4}{|l|}{ De 1979 a $1987^{a}$} \\
\hline Accidentales & E830, E832 & & \\
\hline Mientras esquiaba en el agua & E9100 & 22 & 0,03 \\
\hline \multicolumn{4}{|l|}{ Mientras realizaba otra actividad deportiva } \\
\hline o recreacional con equipo de buceo & E9101 & 39 & 0,05 \\
\hline \multicolumn{4}{|l|}{ Mientras realizaba otra actividad deportiva } \\
\hline o recreacional sin equipo de buceo & E9102 & 440 & 0,53 \\
\hline Nadando o buceando, no deporte & E9103 & 69 & 0,08 \\
\hline En tina de baño & E9104 & 45 & 0,05 \\
\hline Otra especificada & E9108 & 60744 & 73,33 \\
\hline No especificada & E9109 & 9179 & 11,08 \\
\hline Suicidios & E954 & 330 & 0,40 \\
\hline Homicidios & E964 & 4904 & 5,92 \\
\hline Se ignora intensión & E984 & 6427 & 7,76 \\
\hline Total & & 82835 & 100,00 \\
\hline \multicolumn{4}{|l|}{ De 1998 a $2005^{b}$} \\
\hline Accidentales & V90, V92 & & \\
\hline En bañera & W65 & 18 & 0,07 \\
\hline Caída en bañera & W66 & 32 & 0,13 \\
\hline En piscina & W67 & 223 & 0,91 \\
\hline Caída en piscina & W68 & 144 & 0,59 \\
\hline En aguas naturales & W69 & 4202 & 17,16 \\
\hline Caída en aguas naturales & W70 & 1367 & 5,58 \\
\hline Otra especificada & W73 & 2380 & 9,72 \\
\hline No especificada & W74 & 11786 & 48,14 \\
\hline Suicidio & $\mathrm{X} 71$ & 164 & 0,67 \\
\hline Homicidio & X92 & 1532 & 6,26 \\
\hline Se ignora intención & Y21 & 2388 & 9,75 \\
\hline Total & & 24484 & 100,00 \\
\hline Total de 1979 a 2005 & & 107319 & \\
\hline
\end{tabular}

a Según la Clasificación Estadística Internacional de Enfermedades, Traumatismos y Causas de Defunción, 9. ${ }^{a}$ revisión (8). b Según la Clasificación Estadística Internacional de Enfermedades y Problemas Relacionados con la Salud, 10. revisión (9).

FIGURA 1. Tendencia de la mortalidad estandarizada a consecuencia de asfixia por inmersión registrada en México, 1979-2005

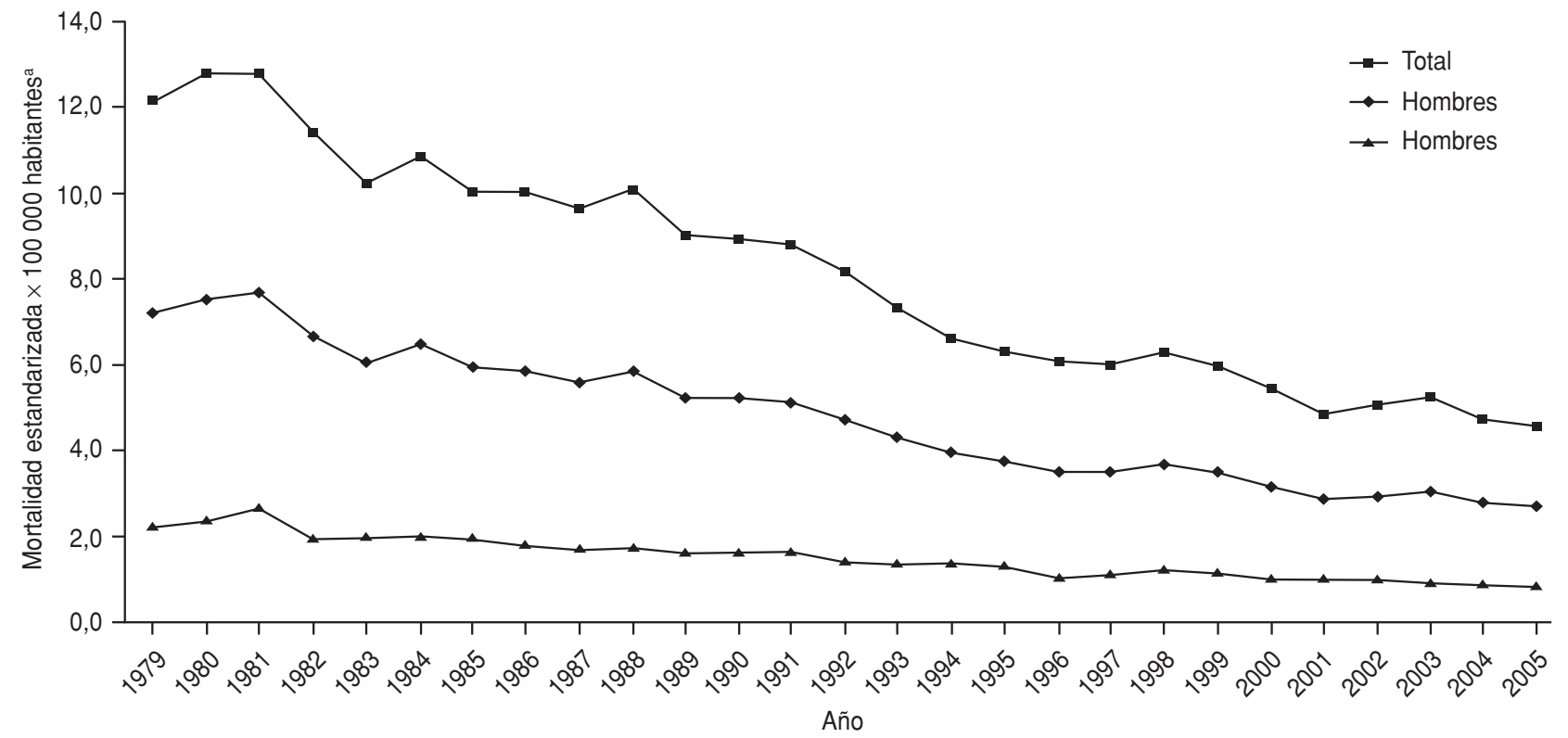

Fuente: Elaborado a partir del análisis de los certificados de defunción de la Secretaría de Salud de México (2).

a Tasa estandarizada según la población de México en el año 2000. 
CUADRO 2. Tasas de mortalidad a consecuencia de asfixia por inmersión, estandarizadas por grupo de edad, ${ }^{\mathrm{a}}$ según el estado federativo. México, 1979-2005

\begin{tabular}{|c|c|c|c|c|c|c|c|c|c|}
\hline Estado & $1979-1981$ & $1982-1984$ & 1985-1987 & $1988-1990$ & 1991-1993 & 1994-1996 & 1997-1999 & 2000-2002 & 2003-2005 \\
\hline Aguascalientes & 5,15 & 4,11 & 3,81 & 4,29 & 2,79 & 2,18 & 2,17 & 1,90 & 1,73 \\
\hline Baja California & 9,79 & 4,95 & 4,42 & 4,81 & 4,36 & 3,52 & 3,43 & 2,55 & 2,72 \\
\hline Baja California Sur & 10,36 & 3,40 & 2,95 & 4,65 & 2,97 & 2,07 & 2,68 & 1,70 & 3,44 \\
\hline Campeche & 14,41 & 7,07 & 5,44 & 8,20 & 5,05 & 4,70 & 5,26 & 4,22 & 2,88 \\
\hline Chiapas & 8,20 & 3,78 & 4,43 & 6,21 & 4,98 & 5,05 & 5,75 & 4,52 & 3,84 \\
\hline Chihuahua & 4,88 & 3,50 & 4,17 & 4,63 & 3,89 & 3,22 & 2,45 & 2,10 & 1,47 \\
\hline Coahuila & 4,46 & 2,50 & 3,32 & 3,24 & 2,83 & 1,85 & 2,14 & 1,44 & 1,60 \\
\hline Colima & 12,91 & 7,23 & 4,63 & 6,32 & 5,81 & 3,04 & 3,96 & 3,79 & 1,65 \\
\hline Distrito Federal & 2,12 & 2,00 & 1,64 & 1,69 & 1,68 & 1,21 & 1,19 & 0,92 & 0,82 \\
\hline Durango & 5,42 & 4,78 & 3,29 & 3,75 & 4,45 & 3,32 & 2,49 & 1,85 & 2,11 \\
\hline Guanajuato & 6,92 & 4,67 & 4,89 & 5,34 & 4,29 & 2,89 & 2,81 & 2,74 & 2,52 \\
\hline Guerrero & 6,88 & 3,92 & 3,40 & 3,67 & 3,99 & 3,21 & 2,86 & 2,31 & 1,97 \\
\hline Hidalgo & 10,81 & 6,69 & 5,00 & 6,81 & 4,82 & 4,33 & 4,17 & 2,77 & 2,68 \\
\hline Jalisco & 4,93 & 3,81 & 3,71 & 4,29 & 4,05 & 3,07 & 2,63 & 2,16 & 2,45 \\
\hline México & 6,22 & 3,39 & 3,20 & 4,13 & 3,53 & 2,64 & 2,34 & 1,97 & 1,66 \\
\hline Michoacán & 5,87 & 4,38 & 4,00 & 4,02 & 3,27 & 3,53 & 3,03 & 2,71 & 2,74 \\
\hline Morelos & 7,99 & 3,70 & 3,49 & 3,92 & 3,37 & 2,64 & 1,98 & 1,99 & 1,94 \\
\hline Nayarit & 5,69 & 5,25 & 4,43 & 6,29 & 5,06 & 4,14 & 2,66 & 3,14 & 3,50 \\
\hline Nuevo León & 3,20 & 1,78 & 1,90 & 4,05 & 2,20 & 1,51 & 1,22 & 1,16 & 0,89 \\
\hline Oaxaca & 7,06 & 5,29 & 4,43 & 4,97 & 4,33 & 4,52 & 4,81 & 3,65 & 3,50 \\
\hline Puebla & 6,81 & 4,76 & 3,76 & 3,62 & 3,12 & 2,63 & 2,82 & 2,06 & 1,66 \\
\hline Querétaro & 8,96 & 4,09 & 4,80 & 4,66 & 4,20 & 2,76 & 3,38 & 2,74 & 2,39 \\
\hline Quintana Roo & 16,07 & 7,02 & 3,75 & 8,30 & 6,21 & 4,51 & 3,44 & 3,02 & 2,37 \\
\hline San Luis Potosí & 7,22 & 5,23 & 5,85 & 5,54 & 4,56 & 3,60 & 3,01 & 2,72 & 2,36 \\
\hline Sinaloa & 9,67 & 8,25 & 6,73 & 5,64 & 5,35 & 4,18 & 4,43 & 3,26 & 3,28 \\
\hline Sonora & 9,26 & 6,29 & 5,98 & 5,52 & 4,68 & 3,68 & 2,85 & 2,90 & 2,27 \\
\hline Tabasco & 24,87 & 12,71 & 9,34 & 13,78 & 11,16 & 9,73 & 8,66 & 7,50 & 5,92 \\
\hline Tamaulipas & 10,50 & 7,20 & 7,88 & 8,39 & 9,01 & 5,67 & 4,42 & 3,54 & 3,54 \\
\hline Tlaxcala & 3,35 & 3,78 & 2,48 & 3,30 & 2,82 & 2,30 & 2,13 & 1,31 & 1,15 \\
\hline Veracruz & 8,92 & 6,46 & 4,90 & 6,63 & 5,62 & 4,23 & 4,31 & 3,98 & 3,32 \\
\hline Yucatán & 3,47 & 1,97 & 2,41 & 3,93 & 2,58 & 2,50 & 2,30 & 1,70 & 1,83 \\
\hline Zacatecas & 5,03 & 4,72 & 3,48 & 3,84 & 3,80 & 3,08 & 1,98 & 2,29 & 1,84 \\
\hline Nacional & 6,58 & 4,43 & 4,00 & 4,71 & 4,05 & 3,23 & 3,02 & 2,53 & 2,28 \\
\hline
\end{tabular}

Fuente: Elaborado a partir del análisis de los certificados de defunción de la Secretaría de Salud de México (2).

a Tasas por 100000 de habitantes, estandarizadas por grupo de edad según la población de México en el año 2000.

\section{DISCUSIÓN}

En el período estudiado, la mortalidad a consecuencia de asfixia por inmersión en México ha mostrado una tendencia descendente, observada en todos los grupos de edad y en todos los estados federativos. Esta tendencia descendente ya ha sido descrita en otros países, aunque en ninguno de América Latina. En Japón, la mortalidad por esta causa en niños de 1 a 4 años disminuyó de 45,4 por 100000 habitantes en 1955 a 1,6 por 100000 habitantes en el año 2000, una reducción de $96,5 \%$ en 45 años (18). En los Estados Unidos, la reducción de la mortalidad a consecuencia de asfixias por inmersión (no asociadas con nau- fragios o caídas de embarcaciones) en menores de 20 años entre los períodos 1971-1973 y 1986-1988 fue de $44,5 \%$ (19). No obstante, las tendencias en los diferentes grupos de edad fueron diversas, ya que la tasa de mortalidad por esta causa se incrementó en $23,2 \%$ en los menores de 1 año (20). En Canadá, la disminución en menores de 15 años entre 1979 y 2002 fue de aproximadamente $66,7 \%$, sin que se observaran cambios considerables en los menores de 1 año (21). En Lituania también se ha informado una tendencia descendente de $66,4 \%$ en niños de 0 a 14 años y de $64,6 \%$ en las personas de 15 a 19 años (22).

Las muertes debidas a asfixia por inmersión se presentaron con mayor fre- cuencia en niños y niñas de 1 a 4 años de edad; en el trienio 2003-2005 se observó la mayor tasa en este grupo de edad en México (4,2 por 100000 habitantes). Si bien estos resultados concuerdan con lo publicado por otros autores $(21,23,24)$, un hallazgo de este estudio fue la identificación de las personas de 60 años o más como el segundo grupo más vulnerable, con una tasa de 3,37 en el período 2003-2005.

Los resultados de esta investigación indican que el tamaño de la población está asociado con la mortalidad a consecuencia de asfixia por inmersión: cuánto más pequeña es la localidad mayor es el riesgo de morir por esta causa. Este resultado coincide con lo encontrado por Fang y colaboradores 
CUADRO 3. Tasas de mortalidad a consecuencia de asfixia por inmersión, según el grupo de edad y el sexo. México, en tres trienios seleccionados

\begin{tabular}{|c|c|c|c|c|c|c|c|c|c|}
\hline \multirow[b]{2}{*}{ Grupo de edad (años) } & \multicolumn{3}{|c|}{ Hombres y niños } & \multicolumn{3}{|c|}{ Mujeres y niñas } & \multicolumn{3}{|c|}{ Total } \\
\hline & No. & Tasa $^{a}$ & IC95\% ${ }^{b}$ & No. & Tasa & IC95\% & No. & Tasa & IC95\% \\
\hline \multicolumn{10}{|l|}{ Período 1979-1981 } \\
\hline De 1 a 4 & 1381 & 11,99 & $11,36-12,63$ & 752 & 6,53 & $6,06-7,00$ & 2143 & 9,35 & $8,96-9,75$ \\
\hline De 5 a 9 & 922 & 5,94 & $5,56-6,32$ & 351 & 2,26 & $2,02-2,50$ & 1286 & 4,17 & $3,94-4,40$ \\
\hline De 10 a 19 & 3261 & 13,01 & $12,57-13,46$ & 705 & 2,81 & $2,61-3,02$ & 3999 & 7,96 & $7,71-8,20$ \\
\hline$\geq 60$ & 786 & 14,95 & $13,91-16,00$ & 140 & 2,66 & $2,22-3,10$ & 933 & 8,46 & $7,92-9,00$ \\
\hline Total & 12638 & 12,76 & $12,53-12,98$ & 2662 & 2,68 & $2,58-2,78$ & 15434 & 7,70 & $7,57-7,82$ \\
\hline \multicolumn{10}{|l|}{ Período 1991-1993 } \\
\hline$<1$ & 90 & 3,02 & $2,39-3,64$ & 72 & 2,41 & $1,86-2,97$ & 164 & 2,79 & $2,36-3,22$ \\
\hline De 1 a 4 & 1105 & 8,60 & $8,09-9,11$ & 615 & 4,79 & $4,41-5,17$ & 1723 & 6,80 & $6,48-7,12$ \\
\hline De 5 a 9 & 550 & 3,41 & $3,13-3,70$ & 211 & 1,31 & $1,13-1,49$ & 764 & 2,38 & $2,21-2,55$ \\
\hline De 10 a 19 & 2425 & 7,94 & $7,63-8,26$ & 478 & 1,57 & $1,43-1,71$ & 2903 & 4,75 & $4,58-4,93$ \\
\hline \multicolumn{10}{|l|}{ Período 2003-2005 } \\
\hline$<1$ & 52 & 1,62 & $1,18-2,06$ & 32 & 1,00 & $0,65-1,34$ & 84 & 1,47 & $1,15-1,78$ \\
\hline De 1 a 4 & 706 & 5,48 & $5,08-5,89$ & 364 & 2,83 & $2,54-3,12$ & 1072 & 4,27 & $4,01-4,52$ \\
\hline De 5 a 9 & 304 & 1,74 & $1,55-1,94$ & 121 & 0,69 & $0,57-0,82$ & 425 & 1,33 & $1,20-1,46$ \\
\hline De 10 a 19 & 1435 & 4,65 & $4,41-4,89$ & 308 & 1,00 & $0,89-1,11$ & 1743 & 2,77 & $2,64-2,90$ \\
\hline De 20 a 39 & 2492 & 5,57 & $5,35-5,79$ & 240 & 0,54 & $0,47-0,60$ & 2735 & 2,86 & $2,76-2,97$ \\
\hline De 40 a 59 & 1230 & 5,12 & $4,83-5,41$ & 125 & 0,52 & $0,43-0,61$ & 1355 & 2,56 & $2,43-2,70$ \\
\hline$\geq 60$ & 712 & 6,59 & $6,11-7,08$ & 101 & 0,94 & $0,75-1,12$ & 814 & 3,37 & $3,13-3,60$ \\
\hline Total & 7108 & 1,62 & $1,18-2,06$ & 1301 & 0,90 & $0,85-0,95$ & 8422 & 2,75 & $2,69-2,81$ \\
\hline
\end{tabular}

Fuente: Elaborado a partir del análisis de los certificados de defunción de la Secretaría de Salud de México (2).

a Tasa por 100000 habitantes.

b IC95\%: intervalo de confianza de 95\%.

en la ciudad de Xiamen y sus alrededores, en la República Popular China, donde la mortalidad en la población de 1 a 14 años de edad en áreas rurales fue 7,8 veces la observada entre los habitantes de áreas urbanas en el mismo grupo de edad (25). La diferencia podría explicarse por la mayor exposición a depósitos de agua naturales (canales, ríos, presas, lagos, etc.) o artificiales (pilas, cisternas, tambos, entre otros).

La distribución geográfica de las defunciones registradas entre 2001 y 2003 muestra un patrón definido, con frecuencias más elevadas en la mayoría de los estados federativos que tienen costas en el Golfo de México (Tabasco, Tamaulipas, Veracruz y Campeche) y en algunos estados con costas en el Océano Pacifico (Baja California Sur,
Nayarit, Oaxaca, Chiapas y Sinaloa). Esta mayor mortalidad no se podría explicar por la vocación turística de esos estados, ya que el análisis de la mortalidad se realizó solo en mexicanos y no según el lugar de defunción sino por el de residencia. Sin embargo, llama la atención la gran diferencia que existe entre la tasa de asfixias por inmersión en el trienio 2003-2005 en Tabasco (5,92 por 100000 habitantes) y el estado que ocupa el segundo lugar nacional (Chiapas: 3,84 por 100000 habitantes). También llama la atención que a pesar de que Tabasco mantuvo esta gran diferencia en la mortalidad por ahogamiento en todos los trienios estudiados, esto no haya sido objeto de un mayor y más profundo análisis.

En Japón, la declinación en la mortalidad se ha atribuido a ciertas modi- ficaciones en el medio ambiente que disminuyeron la exposición y a la educación en salud que redujo el número de muertes por inmersión en tinas de baño, aunque no se han mostrado evidencias científicas que apoyen esta conclusión (18). En Lituania se destacan los cambios en la atención médica, las medidas de prevención de accidentes y las mejoras socioeconómicas recientes, aunque no se señalan acciones específicas dirigidas a disminuir la frecuencia de las muertes por inmersión (22). En el caso de los Estados Unidos, tampoco existe una explicación clara del decremento observado $(19,20)$.

El riesgo de cometer una falacia ecológica por la falta de datos complementarios que brinden una mayor claridad sobre las causas externas y las circunstancias en que ocurrieron estas 
FIGURA 2. Tasa de mortalidad $^{\mathrm{a}}$ a consecuencia de asfixia por inmersión según el estado federativo, México, 2003-2005

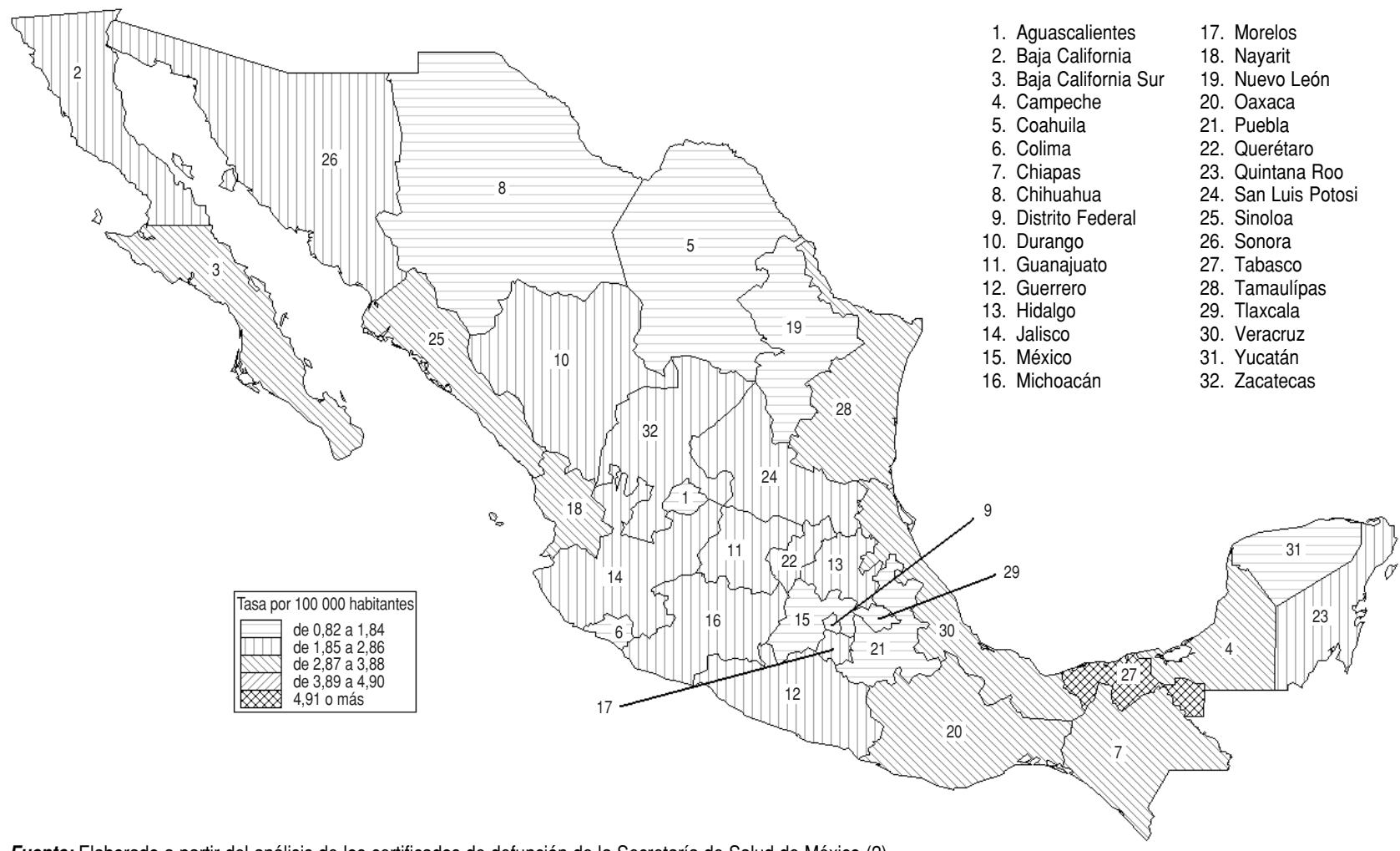

Fuente: Elaborado a partir del análisis de los certificados de defunción de la Secretaría de Salud de México (2).

a Tasa por 100000 habitantes, estandarizada según la población de México en el año 2000.

CUADRO 4. Mortalidad a consecuencia de asfixia por inmersión, según el tamaño de la localidad. México, 2003-2005

\begin{tabular}{lcccc}
\hline $\begin{array}{c}\text { Tamaño de la } \\
\text { localidad, habitantes }\end{array}$ & No. & $\begin{array}{c}\text { Tasa por } 100 \text { 000 } \\
\text { habitantes }\end{array}$ & RR $^{\mathrm{a}}$ & IC95\% $^{\mathrm{b}}$ \\
\hline De 1 a 2 499 & 2945 & 4,03 & 2,15 & 2,05 a 2,26 \\
De 2 500 a 19 999 & 1505 & 3,17 & 1,69 & 1,59 a 1,80 \\
$\geq 20$ 000 & 3483 & 1,87 & 1,00 & \\
\hline
\end{tabular}

Fuente: Elaborado a partir del análisis de los certificados de defunción de la Secretaría de Salud de México (2).

${ }^{\mathrm{a}} \mathrm{RR}$ : riesgo relativo.

b IC95\%: intervalo de confianza de $95 \%$.

muertes impide adelantar alguna hipótesis que describa satisfactoriamente la reducción observada en la mortalidad a consecuencia de asfixia por inmersión en México. Dado que la mortalidad por esta causa en las comunidades rurales es mayor que en las urbanas, una posible explicación de esta reducción estaría asociada con las características del crecimiento demográfico de las últimas décadas, dirigida principalmente hacia comunidades de 20000 habitantes o más y de 2500 a 19999 $(40,0 \%$ y $23,2 \%$, respectivamente, entre 1990 y 2005, mientras las poblaciones de menos de 2500 apenas crecieron en $4,2 \%$ en ese lapso). Sin embargo, los datos analizados - aunque suficientes para hacer una caracterización epidemiológica de la tendencia de la mor- talidad a consecuencia de asfixia por inmersión- no permiten ir más allá en este análisis. En la revisión bibliográfica no se encontró ninguna referencia sobre programas específicos, ya fueran de cobertura nacional o estatal, implementados en México para prevenir estas muertes accidentales.

Durante el período estudiado, en México se cambió el sistema de codificación de las causas de muerte, de la CIE-9 a la CIE-10. Se ha descrito que este cambio puede afectar a la contabilidad de algunas enfermedades, como la cirrosis hepática en la que el registro de muertes aumentó en 3,67\% (26); sin embargo, en esos casos la pendiente de la tendencia muestra cambios que asemejan "escalones" únicos en los años en que se cambió el sistema de clasificación. La tendencia observada en México no muestra que entre 1997 y 1998 se registrara un incremento o disminución sistemática en la mortalidad a 
consecuencia de asfixia por inmersión en ninguna de las categorías estudiadas (ya sea por edad, sexo, tamaño de la localidad, estado de residencia o total). Estos escalones en las tendencias tampoco se observan en trabajos similares de otros autores $(21,22)$. Por ello se puede afirmar que el cambio en el sistema de clasificación de la mortalidad no tuvo un impacto en la tendencia de la mortalidad a consecuencia de asfixia por inmersión en México.

Los estudios de mortalidad basados en datos de defunción tienen algunas fortalezas que deben destacarse. Entre las más importantes se encuentra la posibilidad de tener una cobertura nacional y la consistencia en su sistema de codificación. Estos atributos constituyen la base fundamental de los estudios de tendencia de la mortalidad.
Sin embargo, este estudio también presenta limitaciones que se deben tomar en cuenta al analizar sus resultados. En primer lugar, al estar orientado a la mortalidad no atiende una de las facetas de este problema de salud: la morbilidad, de la que se sabe muy poco en México y en la mayoría de los países con ingresos medios y bajos. Además, el número de variables que se puede estudiar a partir de los certificados de defunción en México es limitado y la inestable calidad de esos datos puede afectar a la validez de estos resultados. A pesar de que en México la certificación de las muertes generalmente está a cargo de un médico legista, lo que reduce las fallas en los datos de origen, una gran parte de las defunciones no estaba bien clasificada (más de $55 \%$ se clasificaron como "Otra especificada" o
"No especificada" y los únicos cuerpos de agua especificados correspondieron a tinas de baño y piscinas). Esto limita aún más las posibilidades de análisis.

A pesar de estas limitaciones, en este trabajo se demostró que la mortalidad a consecuencia de asfixia por inmersión en México presenta una tendencia descendente, a pesar de lo cual se mantiene como un importante problema de salud pública. Es necesario profundizar en la consolidación de la información sobre las muertes accidentales por esta causa, tanto desde el punto de vista de la vigilancia epidemiológica como de las intervenciones que se deben implementar para su erradicación. Se debe establecer un registro de cobertura nacional que abarque tanto la información sobre la mortalidad como sobre la morbilidad de la asfixia por inmersión.

\section{REFERENCIAS}

1. Peden MM, McGee K. The epidemiology of drowning worldwide. Int J Inj Contr Saf Promot. 2003;10(4):195-9.

2. Sistema Nacional de Información en Salud. Información estadística. Mortalidad. México, D.F.: Secretaría de Salud; 2007. Hallado en http://sinais.salud.gob.mx/mortalidad/. Acceso el 4 de agosto de 2008.

3. Celis A, Gómez-Lomelí Z, Armas J. Tendencias de mortalidad por traumatismos y envenenamientos en adolescentes. Salud Publica Mex. 2003;45(Supl1):8-15.

4. Celis A. Asfixia por inmersión en Jalisco, 1983-1989. Salud Publica Mex. 1991;33(6): 585-9.

5. Celis A. Home drowning among preschool age Mexican children. Inj Prev. 1997;3(4): 252-6.

6. Sapkota S, Kohl HW, Gilchrist J, McAuliffe J, Parks B, England B, et al. Unauthorized border crossings and migrant deaths: Arizona, New Mexico, and El Paso, Texas, 2002-2003. Am J Public Health. 2005;96(7):1282-7.

7. Guptill KS, Hargarten SW, Baker TD. American travels deaths in Mexico. Causes and prevention strategies. West J Med. 1991;154(2): $169-71$.

8. Organización Mundial de la Salud. Clasificación Estadística Internacional de Enfermedades, Traumatismos y Causas de Defunción. 9. ${ }^{\text {a }}$ revisión. Washington, D.C.: Organización Panamericana de la Salud; 1978.

9. Organización Mundial de la Salud. Clasificación Estadística Internacional de Enfermedades y Problemas Relacionados con la Salud. 10. ${ }^{\mathrm{a}}$ revisión. Washington, D.C.: Organización Panamericana de la Salud; 1998.
10. Rotman K, Greenland S. Modern epidemiology. 2. ${ }^{\text {a }}$ ed. Filadelfia: Lippincott-Raven; 1998.

11. Instituto Nacional de Estadísticas, Geografía e Informática. Censo General de Población y Vivienda, 2000 [CD Rom]. Aguascalientes, México: INEGI; 2001

12. Camel F. Estadística médica y planificación de la salud. Vol II. Mérida, Venezuela: Consejo de Publicaciones de la Universidad de los Andes; 1982.

13. Instituto Nacional de Estadísticas, Geografía e Informática. Censo General de Población y Vivienda, 1980. México, D.F.: INEGI; 1984.

14. Instituto Nacional de Estadísticas, Geografía e Informática. XI Censo General de Población y Vivienda, 1990. Resumen general. Aguascalientes, México: INEGI; 1992

15. Instituto Nacional de Estadísticas, Geografía e Informática. Conteo de Población y Vivienda, 1995. Resultados definitivos. Tabulados básicos. Aguascalientes, México: INEGI; 1997.

16. Instituto Nacional de Estadísticas, Geografía e Informática. Conteo de Población y Vivienda, 2005 [sitio en Internet]. Aguascalientes, México: INEGI; 2006. Hallado en: http://www. inegi.gob.mx/inegi $/$ default.aspx? $\mathrm{s}=\mathrm{est} \& \mathrm{c}=$ 10215. Acceso el 4 de agosto de 2008.

17. Dean AG, Arner TG, Sangam S, Sunki GG, Friedman R, Lantinga M, et al. Epi Info 2000, a database and statistics program for public health professionals for use on Windows 95, 98, NT, and 2000 computers. Atlanta, GA: Centers for Disease Control and Prevention; 2000.

18. Nakahara S, Ichikawa M, Wakai S. Drowning deaths among Japanese children aged 1-4 years: different trend due to different risk reductions. Inj Prev. 2004;10(2):125-6.
19. Smith G, Howland J. Declines in drowning: exploring the epidemiology of favorable trends. J Am Med Assoc. 1999;281(23):2245-7.

20. Brenner RA, Smith GS, Overpeck MD. Divergent trends in childhood drowning rates, 1971 through 1988. J Am Med Assoc. 1994;271(20): 1606-8.

21. Pan SY, Ugnat AM, Semenciw R, Desmeules M, Mao Y, Macleod M. Trends in childhood injury mortality in Canada, 1979-2002. Inj Prev. 2006;12(3):155-60.

22. Strukcinskiene B. Unintentional injury mortality trends in children and adolescents in Lithuania between 1971 and 2005. Int J Inj Contr Saf Promot. 2008;15(1):1-8.

23. Brenner RA. Prevention of drowing in infants, children and adolescent. Pediatrics. 2003; 112(2):440-5.

24. Yang L, Nong QQ, Li CL, Feng QM, Lo SK. Risk factors for childhood drowning in rural regions of a developing country: a case-control study. Inj Prev. 2007;13(3):178-82.

25. Fang Y, Dai L, Jaung MS, Chen X, Yu S, Xiang $H$. Child drowning deaths in Xiamen city and suburbs, People's Republic of China, 20012005. Inj Prev. 2007;13(5):339-43.

26. Yoon $Y, Y i$ H. Liver cirrhosis mortality in the United States, 1970-2003. Arlington, VA: National Institute on Alcohol Abuse and Alcoholism; 2006. (Surveillance Report No. 75.)

Manuscrito recibido el 11 de diciembre de 2007. Aceptado para publicación, tras revisión, el 11 de agosto de 2008. 
ABSTRACT Objective. To examine the trends in mortality from drowning in Mexico and each of its federal states during the period from 1979-2005.

\section{Trends in mortality from drowning, Mexico, 1979-2005}

Methods. Mortality rates and trends were estimated for the study period from Mexico's official data based on the International Classification of Diseases, 9th and 10th revisions. Results were analyzed by age group, sex, and year registered, as well as by state and size of city or town of residence.

Results. From 1979-2005, there were 107319 deaths by drowning registered. The mortality from drowning rate decreased $65.31 \%$ (from 6.58 per 100000 inhabitants in 1979-1981 to 2.28 per 100000 inhabitants in 2003-2005). This decrease was evident in all the age groups (ranging from $68.10 \%$ among $5-9$ year olds to $54.30 \%$ among $1-4$ years) and by state (ranging from $87.19 \%$ in Colima to $36.58 \%$ in Nayarit). During the three-year period from 2003-2005, drowning mortality was relatively greater among men and boys than among women and girls (rate: 5.46 (95\%CI: 5.15-5.80); among the age groups $1-4$ years (4.27 per 100000$)$ and 60 years or older (3.37 per 100000 inhabitants); in the Tabasco state (5.92 per 100000 inhabitants); and among towns with fewer than 2499 inhabitants (4.03\% per 100000 inhabitants).

Conclusions. In Mexico, the drowning mortality drowning rate is on a downward trend, although it remains a serious public health problem. Intensified efforts are needed to harness information regarding accidentals death from this cause, both from the epidemiological surveillance angle as well as through the implementation of eradicating interventions.

Key words Drowning, mortality, Mexico. 